\title{
BRITISH SOCIETY OF
}

\section{ANIMAL PRODUCTION}

\section{Report of Proceedings}

of the Eleventh Meeting, 3rd March 1949

General Topic:

INCREASING BEEF PRODUCTION IN BRITAIN

\section{PUBLISHED BY THE SOCIETY}

Price 3/- 


\section{CONTENTS}

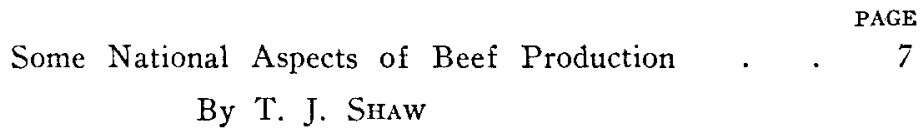

Beef from Dairy and Dual purpose Calves . . . 21 By W. S. Mansfield

Discussion . . . . . . . . . 27

Raising Beef Cattle on the Welsh Hills . . . $\quad 34$ By Capt. Bennett Evans

Store Cattle from the Scottish Hills . . . . 41 By D. Stewart

Discussion . . . . . . . . . 47 


\title{
EXECUTIVE COMMITTEE, 1949
}

\author{
President: Mr. E. C. J. Allday. \\ Vice-President: Mr. J. R. Currie.
}

Professor T. L. Bywater.

Mr. H. R. Davidson.

Mr. J. Edwards.

Dr. A. B. FoWler.

Mr. Moses Griffith.

Dr. J. Hammond.

Mr. A. Hobson.

Professor W. C. Miller.

Professor J. E. Nichols.

Professor J. A. Scott Watson.

Professor R. G. White.

Secretary-Treasurer: Mr. J. P. Maule.

Commonwealth Bureat of Animal

Breeding and Genetics,

King's Buildings,

Edinburgh, Scotland. 